\title{
Cell constant studies of bipolar and tetrapolar electrode systems for impedance measurement
}

\author{
Hanbin $\mathrm{Ma}^{\mathrm{a}}$, Yang $\mathrm{Su}^{\mathrm{a}}$, Arokia Nathan ${ }^{\mathrm{a}^{*}}$ \\ ${ }^{\text {a }}$ Electrical Division, Engineering Department, University of Cambridge, 9 JJ Thomson \\ Avenue, Cambridge, CB3 OFA, U.K. \\ * To whom correspondence should be addressed: Tel: +44 (0) 1223 748302, Fax: +44 (0) \\ 1223748322 \\ E-mail:an299@cam.ac.uk
}

\section{Keywords}

bipolar and tetrapolar electrodes, impedance sensor, planar thin-film electrodes, cell constant, electrode polarization.

\section{Highlights}

- Method to calculate cell constant in impedance measurement systems.

- Cell constant used to evaluate performance of planar electrode systems.

- Planar electrodes fabricated using large-area processes.

- FEM used to simulate the performance of planar electrodes.

- Concentration tests with bipolar and tetrapolar electrode systems and quantitative assessment of performance. 


\begin{abstract}
In comparison to the bipolar or two-electrode system, the tetrapolar or four-electrode arrangement is a well-known technique to reduce electrode polarization just as effectively. However, recent studies have showed that phenomena such as negative sensitivity and multiple current paths can compromise the advantages of the tetrapolar electrode arrangement, thereby potentially limiting its applications. This paper reveals a novel method to evaluate the performance of the different electrode systems in which the concept of cell constant is extended to an impedance measurement system. We employ it as a standardised parameter to quantitatively analyse planar electrode systems in bipolar and tetrapolar measurement modes. Indeed, the cell constant is a key parameter in conductivity sensors to evaluate electrodes designs since it is independent of any readout electronics. A comparison of measurement modes using finite element methods (FEM) simulations and measurements for sodium chloride solutions is presented. While the cell constant of the bipolar electrode system is one order of magnitude greater than that of the tetrapolar arrangement, it shows large discrepancy over the measured frequency range. In contrast, despite the existence of measurement errors, the tetrapolar arrangement yields a uniform cell constant and good agreement with the simulations.
\end{abstract}




\section{Introduction}

A typical biochemical sensing system can be mainly divided into three parts. A sampleunder-tested (SUT) part which can be either in the form of solid, liquid or gas, containing the analyte/analytes to be sensed. A signal processing part is used for signal amplification and data analysis. And finally a transducer, interfacing the other two parts, is used to convert the sample output (in any form) to a readable signal for the processing part [1]. Each of the three parts would affect the final sensing performance (e.g. sensitivity, detection limit, etc.). Specific considerations have to be taken for each part when designing a sensing system in order to achieve the best overall performance. For instance, pre-measurement sample handling is normally required for most biosensing system to minimise the interference of native samples [2]. The latest technology node (e.g. the different CMOS technology) always tends to be selected to maximize the capabilities of the signal processing part [3]. Optimised transducer design could enhance the sensing capabilities significantly [4].

Electrodes are employed as the transducer for a wide range of biochemical sensors. For example, electrodes functionalization is one of the common ways to improve the selectivity and the detection limit [5-7], while the optimisation of the geometry is a good approach to improving the sensitivity [8,9]. Large-area processing technologies provide the means to manufacture thin-film planar electrode systems at low cost with a large degree of design freedom [10-13]. The sensors' properties, such as sensitivity and dynamic range, can be simply fine-tuned by varying the electrode dimensions $[14,15]$ to suit different requirements. Here, a standardised parameter which is independent of 
readout electronics and one that purely reflects the performance of electrodes is highly desirable.

The cell constant of an electrolyte conductivity sensor, defined as the ratio of the measured resistance and the specific resistance of electrolyte, turns out to be an effective parameter to evaluate the electrode performance. Here the cell constant is linear and inversely proportional to the sensitivity [16]. The concept has been extended to capacitance-based sensing [17], and hence would be applicable for impedance-based measurements as well $[18,19]$.

Conventional impedance-based measurements use a bipolar electrode arrangement, in which the same electrode pair is shared for both current carrying (CC) and voltage pick up (PU). However, an electrolyte-electrode double layer is formed by the driving electric field near the CC surfaces; also known as electrode polarization [20]. A tetrapolar electrode arrangement separates the PU from CC, and may effectively eliminate the electrode polarization [21] at the expense of introducing additional interferences embracing negative sensitivity regions and separate current paths [22,23]. These measurement errors serve to limit the advantages of the tetrapolar system over the bipolar counterpart.

This paper, reports on the use of cell constant to quantitatively compare the performance of planar bipolar and tetrapolar electrode systems. The method of cell constant calculation for impedance-based systems is described and the performance of bipolar and tetrapolar electrode systems is compared using both finite element methods (FEM) simulation and saline concentration measurements. 


\section{Materials and methods}

2.1 Cell constant of electrolyte impedance sensors

In an electrolyte conductivity sensor, the cell constant $\kappa\left(\mathrm{cm}^{-1}\right)$ is defined as the proportionality between electrolyte resistivity $\rho(\Omega \cdot \mathrm{cm})$ and measured resistance $R(\Omega)$ :

$\kappa=\frac{R}{\rho}=R \cdot \sigma$

where $\sigma(\mathrm{mS} / \mathrm{cm})$ represents the conductivity of the electrolyte.

For a parallel plate electrode system with homogeneous dielectric medium, the resistance $R$ can be calculated as:

$R=\frac{\rho \cdot l}{A}=\frac{l}{\sigma \cdot A}$

where $l$ and $A$ denote the length of the dielectric material and the area of the electrical contacts, respectively. For the same system, the capacitance $C$ can be calculated as:

$C=\frac{\varepsilon_{0} \cdot \varepsilon_{r} \cdot A}{l}$

where $\varepsilon_{0}$ is the vacuum permittivity and $\varepsilon_{r}$ the relative permittivity. Thus,

$R \cdot C=\frac{\varepsilon_{0} \cdot \varepsilon_{r}}{\sigma}$

By combining equation (1) and (4), the cell constant $\kappa$ can be expressed in terms of the measured capacitance as:

$\kappa=\frac{\varepsilon_{0} \cdot \varepsilon_{r}}{C}$ 
Therefore, the total impedance $Z$ of the system with known $R$ and $C$ can be expressed using the cell constant $\kappa$ :

$Y=\frac{1}{Z}=\frac{1}{R}+j \cdot \omega \cdot C=\frac{\sigma+j \cdot \omega \cdot \varepsilon_{0} \cdot \varepsilon_{r}}{\kappa}$

Here, $Y$ denotes the admittance (the inverse of impedance). The magnitude of the impedance $|Z|$ is:

$|Z|=\frac{\kappa}{\sqrt{\sigma^{2}+\omega^{2} \cdot \varepsilon_{0}^{2} \cdot \varepsilon_{r}^{2}}}$

Thus any given impedance circuit model can be expressed by an equivalent resistance in parallel with a capacitance. Equation (7) is applicable to all impedance-based measurement systems.

In addition, by using conformal mapping, any field distribution of a planar electrodes system can be converted to that of a corresponding parallel electrode system [24].

Therefore, the cell constant expression above can be used for all planar electrode systems.

2.2 Bipolar and tetrapolar planar electrode systems

Thin-film metal electrodes were deposited with a thermal evaporator on top of a glass substrate as shown in Fig. 1(a). A $20 \mathrm{~nm}$ Cr layer was applied for adhesion before an 80 $\mathrm{nm}$ Au layer, and patterned using a shadow mask. Both the separation and the width of the electrodes were $0.5 \mathrm{~mm}$ while the length was $4 \mathrm{~mm}$. The electrodes were surrounded by a PDMS ring to contain the electrolyte for impedance measurements.

For the bipolar electrode set-up, only the inner pair electrodes were used during the measurement. The pair functioned as both input current carrying (CC) electrodes and 
output voltage pick up (PU) electrodes as shown in Fig. 1(b). For tetrapolar

measurements, the inner pair was used for PU only while the outer electrodes pair was used for CC.

\subsection{Sodium chloride concentration measurements}

Eight groups of $\mathrm{NaCl}$ solutions with different concentrations were prepared for the impedance measurements as shown in Table 1. The $\mathrm{NaCl}$ was purchased from SigmaAldrich (S7653) and was obtained as white powder. Deionized (DI) water from SigmaAldrich (38796) was used with conductivity lower than $4.3 \mu \mathrm{S} / \mathrm{cm}$. Firstly, $10 \mathrm{~g}$ of $\mathrm{NaCl}$ powder was dissolved in one litre of deionized (DI) water to prepare a stock solution with a concentration of $10 \mathrm{mg} / \mathrm{ml}$. The stock solution was diluted with DI water by 1:1 yielding a concentration of $5 \mathrm{mg} / \mathrm{ml}$, and further diluted by the same ratio for concentration of $2.5 \mathrm{mg} / \mathrm{ml}$. All of the different-concentration samples were prepared according to this dilution process. For the bipolar and tetrapolar measurements, $80 \mu 1$ of each sample was used. The impedance measurements were carried out using Solartron 1260 Gain/Phase Analyzer. An AC signal with the amplitude of $100 \mathrm{mV}$ was used to ensure the linearity of the response [25], and the frequency range was from $100 \mathrm{~Hz}$ to 1 $\mathrm{MHz}$ at room temperature. For each frequency sweep, the measurement time is less than three minutes, and a one second integration time was used between measurements to avoid too much Joule heating in the cell. The linearity of the response of $\mathrm{NaCl}$ solution around $100 \mathrm{mV}$ was confirmed, and the detailed results are shown in Appendix A. Within the selected concentration range $(10 \mathrm{ml} / \mathrm{ml}$ to $0.078 \mathrm{mg} / \mathrm{ml})$, both the conductivity and permittivity of the $\mathrm{NaCl}$ solution were linearly proportional to the solution concentration [26]. Meanwhile, for the same concentration, the solution's electrical properties remained constant within the measurement frequency range $(100 \mathrm{~Hz}$ to $1 \mathrm{MHz})[27,28]$. The values used for simulation and cell constant calculation are listed in Table 1.

\begin{tabular}{ccc}
\hline $\begin{array}{c}\mathrm{NaCl} \text { solution } \\
\text { concentration } \\
(\mathrm{mg} / \mathrm{ml})\end{array}$ & $\begin{array}{c}\text { Approx. conductivity } \\
(\mathrm{S} / \mathrm{m})\end{array}$ & $\begin{array}{c}\text { Approx. relative } \\
\text { permittivity }\end{array}$ \\
\hline 10 & 1.7600 & 76.0 \\
\hline
\end{tabular}




\begin{tabular}{ccc}
\hline 5 & 0.9000 & 78.2 \\
\hline 2.5 & 0.4800 & 79.3 \\
\hline 1.25 & 0.2480 & 79.8 \\
\hline 0.625 & 0.1240 & 80.1 \\
\hline 0.312 & 0.0617 & 80.2 \\
\hline 0.156 & 0.0320 & 80.3 \\
\hline 0.078 & 0.0135 & 80.3 \\
\hline
\end{tabular}

Table 1. Concentrations of $\mathrm{NaCl}$ solution used for measurement and their electrical properties.

\subsection{FEA simulation model}

Finite element analysis (FEA) of the bipolar and tetrapolar electrode systems was performed using COMSOL Multiphysics AC/DC package. A 3-dimensional model (as shown in Fig. 1(a)) was synthesised based on the specifications of the fabricated thin-film electrodes. The model consists of a glass substrate, metal electrodes and conductive electrolyte. A cross sectional view of the model and a cut line (A-A') used for further data analysis is shown in Fig. 1(c) and (d). The electrode dimensions shown are based on the fabricated device.

Fig. 1(c) shows the simulation setup for the bipolar electrode arrangement, in which the inner electrode pair was connected to a current source and outer pair was floating, and the voltage difference between the PU electrodes was recorded for impedance calculations. For the tetrapolar simulation shown in Fig. 1(d), the inner electrode pair was connected to a high $Z$ end to record the voltage only, and the current source was connected to the outer pair. The stimulus frequency was $10 \mathrm{kHz}$, and the input current was $1 \mathrm{~A}$.

\section{Results and discussion}




\subsection{FEA simulation}

In impedance measurements, a source current is injected from the $\mathrm{CC}$ electrodes, leading to an electric field in the electrolyte. The corresponding simulated equal-potential distributions for both bipolar and tetrapolar systems with $0.078 \mathrm{mg} / \mathrm{ml} \mathrm{NaCl}$ solution are shown in Fig. 2(a) and (b). The horizontal and vertical axes denote dimensions of the model and are in units of $\mathrm{nm}$, and the solid line (at $\mathrm{y}=0.5 \times 10^{6} \mathrm{~nm}$ ) indicates the interface between the glass substrate and the electrolyte. Four electrodes are located on this interface, which are labelled in white. A current source of $1 \mathrm{~A}$ is used in the simulations; the color bars indicate the value of the potential in volts.

The polarization distributions of the tetrapolar and bipolar systems are shown in Fig. 2(b) and (c). The color bar indicates the polarization strength in $\mathrm{C} / \mathrm{m}^{2}$. The simulations results from the COMSOL software were subsequently processed by Microsoft VISIO to enhance visibility. Fig. 2(e) shows the polarization values for both bipolar and tetrapolar systems at the cut line A-A'.

The driving source in the impedance measurement system is current. The current leads to the build-up of electric surface charges (in the unit of $\mathrm{C} / \mathrm{m}^{2}$ ) that denotes the electrode polarization [29]. The current density $(J)$ can be stated in terms of electric field $(E)$ and conductivity $(\sigma)$ :

$J=\sigma \cdot E$

The polarization $(P)$ can be expressed by

$$
P=\varepsilon_{0} \cdot \chi_{e} \cdot E \quad \text { or } \quad P=\varepsilon_{0} \cdot\left(\varepsilon_{r}-1\right) \cdot E
$$


where $\chi_{e}$ is the electric susceptibility, which is related to the dielectric permittivity $\left(\varepsilon_{\mathrm{r}}\right)$. By combining equation (8) and (9), we have

$P=\varepsilon_{0} \cdot\left(\varepsilon_{r}-1\right) \cdot \frac{J}{\sigma}$

Therefore, the polarization is directly related to current density. In bipolar measurements, the inner pair of electrodes are shared as both CC and PU. A high local polarization results from the high current density as shown in Fig. 2(c). In contrast, the tetrapolar setup separates CC from PU, and the inner pair electrodes are connected to a high impedance input to read the voltage only. Ideally, there should be zero current flow through the PU with zero surface charge and therefore zero polarization. However, as shown in Fig. 2(e), zero polarization areas can only be observed in the middle region of the PU electrodes, while the edges are still polarised due to edge effects [30]. In this simulation, the mean normalized polarization between PUs (from $X=-0.75 \mathrm{~mm}$ to $\mathrm{X}=0.75$ mm on cutline A-A') is $0.0139 \mathrm{C} / \mathrm{m}^{2}$ for the bipolar system compared to $0.0071 \mathrm{C} / \mathrm{m}^{2}$ for the tetrapolar counterpart. The latter has nearly $50 \%$ less polarization.

However, the AC/DC module of the COMSOL software is only able to simulate the strength of polarization. The polarization related parasitic impedance values cannot be extracted since the electrolyte is considered to be homogenous by the simulator. The simulated impedance only indicates the ideal results of different electrode systems, which will be used as references in later analysis.

\subsection{Impedance measurement}


The magnitude of the impedance for different saline concentrations as a function of frequency is shown in Figure 3. The subplot in Fig. 3(a) depicts a typical equivalent circuit of the bipolar electrode system. The circuit consists of a solution capacitance $\left(C_{s}\right)$, a measured resistance $\left(R_{\text {measured }}\right)$ and a polarization capacitance $\left(C_{p}\right)$. This model is used for data fitting, and the fitted parameters can be found in Appendix B. However, this equivalent circuit model is not suitable for the tetrapolar arrangement. The capacitive elements here are affected by measurement errors and will be explained later in this section, while the resistance, $R_{\text {measured }}$ can be calculated from the plot directly.

As shown in Fig. 3(a), the polarization capacitance of the bipolar arrangement dominates the impedance behaviour at low frequency (from $1 \mathrm{kHz}$ to $100 \mathrm{kHz}$ ) for all eight samples. But as the frequency increases, the resistive element starts to prevail and the approximate polarization cut-off frequency $\left(f_{t}\right)[31]$ :

$f_{t} \approx \frac{1}{2 \pi \cdot R_{\text {measured }} \cdot C_{p}}$

decreases as the measured resistance increases. A higher cut-off frequency (>1 MHz) mainly caused by the solution capacitance $\left(C_{s}\right)$ can also be observed.

The polarization cut-off frequency for the tetrapolar electrode system cannot be observed due to the minimised polarization induced impedance. The impedance of lowconcentration samples is unstable at frequencies below $2 \mathrm{kHz}$ because of parasitic capacitances in the measurement set-up since more wires and connections are needed in order to accommodate more electrodes. 
One of the major concerns of the tetrapolar measurement is the separate current paths caused by the voltage readout electronics of the PU electrodes. Ideally, the input impedance of the PU readout should be infinite to block the current while reading the voltage. However, this cannot be realized in real measuring system. The non-ideal PU readouts lead to separate current paths in the system, which causes the early roll-off of the measured impedance in Fig. 3(b) indicated as 'tetrapolar measurement error' [32].

Figure 4 illustrates the measured and simulated impedance behaviour as a function of concentration at a frequency of $10 \mathrm{kHz}$ for both bipolar and tetrapolar electrode arrangements. We observe that the impedance of the former is consistently higher than that of the tetrapolar counterpart for all concentrations. Again, this is due to measurement errors of the tetrapolar electrode system, which give rise to negative sensitivity zones. These are found between CC and PU, where a lower global impedance is detected while there are increases in the local impedance [22]. Fig. S.3 in Appendix C shows the sensitivity map of the tetrapolar electrode system.

As shown in the log-log plot in Fig. 4, there is an increasing gap between the simulation and measurement results as the concentration of the sample increases for the bipolar electrode system. This is mainly due to the polarization induced impedance which can be divided into polarization induced resistance and capacitance. Polarization induced capacitance is also known as double layer capacitance. A double layer is formed by the charges accumulated on the surface of the electrodes immersed in electrolyte. Two layers of opposite charges will be formed at the interface between the electrodes and the electrolytic solution. The distribution of charge typically obeys Maxwell-Boltzmann equations. The polarization induced capacitance depends on the ionic strength and the 
potential applied on the electrodes. The double layer can be described by the GouyChapman model. In addition to the capacitive element, the double layer also contributes to a resistive element, which is only affected by the ion concentration and is independent of the stimulus frequency.

In Fig 4, the equivalent circuit model-fitted values of $R_{\text {measured }}$ are denoted by the solid squares of the equivalent circuit model. The impedance differences between the modelfitted values and the simulation results indicate the polarization induced resistance, and the differences between the measured results and the model-fitted values denote the polarization induced capacitance.

As shown in Fig.S5 in Appendix D, the polarization impedance reduces gradually as the concentration of the sample increases, while the polarization capacitance remains almost the same. In contrast, the measured data of tetrapolar set-up corroborates with simulations for all concentrations, confirming that the measured polarization induced impedance in the tetrapolar electrode system is negligible. The fitted $R_{\text {measured }}$ also agrees well with the measured results because of the purely resistive nature of the sample at 10 $\mathrm{kHz}$.

\subsection{Cell constant}

The cell constants of both the bipolar and tetrapolar electrode systems are calculated using the measured impedance data along with the relations introduced in Section 2.1 and parameters in Table 1. At each measured frequency, eight samples of different concentrations yield eight cell constants. The mean values are plotted in Fig. 5 with the standard deviation as the error bar. The comparison between the ideal cell constants 
derived from the COMSOL simulations and the calculated values at a frequency of 10 $\mathrm{kHz}$ are shown in the subplot in Fig.5. The measured cell constant of the bipolar electrode system is one order of magnitude larger than that of simulations albeit with a great error bar, while the simulated and measured cell constant values of the tetrapolar electrode system are in good agreement.

The cell constant of the bipolar electrode system decreases as the frequency increases until it reaches the cut-off frequency where all eight samples show purely resistive behaviour. Here the calculated values are dramatically affected by the polarization capacitance at frequencies below $100 \mathrm{kHz}$. The cell constant values are also biased by the polarization resistance over all frequencies. The polarization induced impedance is heavily dependent on the properties of the electrolyte. Thus, the sample-dependent cell constant in the bipolar electrode system is no longer able to evaluate the electrode performance objectively.

On the other hand, the values of the cell constants of the tetrapolar electrode system relatively uniform over the frequency range, $1 \mathrm{kHz}$ to $10 \mathrm{MHz}$. The error bar is also generally small, and the larger discrepancy above $1 \mathrm{MHz}$ is mainly caused by the impedance roll-off associated with the higher cut-off frequency. Since the tetrapolar electrode system is almost free of polarization, the measured cell constants are more than one order lower than the bipolar counterparts. More importantly, the measured cell constant of the tetrapolar setup is concentration-independent, hence can be used to objectively evaluate the electrode performance. 
It is worth mentioning that, the universal applications of bipolar electrode system in determining the cell constants are based on single frequency measurement only. Without considering the frequency-dependent (capacitive) element, the method is only applicable for the conductivity measurement. In our study, we extended the concept of cell constant from the real domain (i.e. purely resistive, $\kappa=\frac{R}{\rho}=R \cdot \sigma$ ) to the complex domain (i.e. both resistive and capacitive, $\left.\kappa=|\mathrm{Z}| \cdot \sqrt{\sigma^{2}+\omega^{2} \cdot \varepsilon_{0}^{2} \cdot \varepsilon_{\mathrm{r}}^{2}}\right)$. The bad results with bipolar electrode system were observed in lower frequency range $(1 \mathrm{kHz}-100 \mathrm{kHz})$. This can only be revealed by the frequency-dependent complex domain cell constant study.

\section{Conclusions}

In this paper, the cell constant is used as a standardised parameter to quantitatively evaluate the performance of planar electrode systems for solutions with different sodium chloride concentrations. The tetrapolar system, which is more stable with higher sensitivity, has been proven to be a better arrangement as compared to the bipolar electrode counterpart. As part of the ongoing work, finite element simulations along with experiments using more complex bio-samples are being carried out to fully validate the evaluation method presented here.

\section{Acknowledgements}

This work is partially supported by the Isaac Newton Trust. 


\section{Reference}

[1] E.A.H. Hall, Biosensors, Open University Press, Buckingham, 1990.

[2] P.R.C. Gascoyne, J. V Vykoukal, Dielectrophoresis-Based Sample Handling in General-Purpose Programmable Diagnostic Instruments., Proc. IEEE. Inst. Electr. Electron. Eng. 92 (2004) 22-42. doi:10.1109/JPROC.2003.820535.

[3] J.M. Rothberg, W. Hinz, T.M. Rearick, J. Schultz, W. Mileski, M. Davey, et al., An integrated semiconductor device enabling non-optical genome sequencing., Nature. 475 (2011) 348-52. doi:10.1038/nature10242.

[4] J. Wang, Electrochemical biosensors: towards point-of-care cancer diagnostics., Biosens. Bioelectron. 21 (2006) 1887-92. doi:10.1016/j.bios.2005.10.027.

[5] G. Ja, E. Gyurcsa, Hybridization-Modulated Ion Fluxes through Peptide-NucleicAcid- Functionalized Gold Nanotubes . A New Approach to Quantitative LabelFree DNA Analysis, (n.d.) 2-5.

[6] J. Liu, S. Tian, P.E. Nielsen, W. Knoll, In situ hybridization of PNA/DNA studied label-free by electrochemical impedance spectroscopy., Chem. Commun. (Camb). (2005) 2969-71. doi:10.1039/b419425j.

[7] T. Lindfors, L. Höfler, G. Jágerszki, R.E. Gyurcsányi, Hyphenated FT-IRattenuated total reflection and electrochemical impedance spectroscopy technique to study the water uptake and potential stability of polymeric solid-contact ionselective electrodes., Anal. Chem. 83 (2011) 4902-8. doi:10.1021/ac200597b.

[8] Q. Liu, J. Yu, L. Xiao, J.C.O. Tang, Y. Zhang, P. Wang, et al., Impedance studies of bio-behavior and chemosensitivity of cancer cells by micro-electrode arrays., Biosens. Bioelectron. 24 (2009) 1305-10. doi:10.1016/j.bios.2008.07.044.

[9] M. Varshney, Y. Li, Interdigitated array microelectrodes based impedance biosensors for detection of bacterial cells., Biosens. Bioelectron. 24 (2009) 295160. doi:10.1016/j.bios.2008.10.001.

[10] C. Smith, H. White, Theory of the voltammetric response of electrodes of submicron dimensions. Violation of electroneutrality in the presence of excess supporting electrolyte, Anal. Chem. (1993) 3343-3353. http://pubs.acs.org/doi/abs/10.1021/ac00071a002 (accessed December 18, 2014).

[11] J. Conyers, H. White, Electrochemical characterization of electrodes with submicrometer dimensions, Anal. Chem. 72 (2000) 4441-4446. http://pubs.acs.org/doi/abs/10.1021/ac000399\%2B (accessed December 18, 2014). 
[12] W. Qu, W. Wlodarski, A thin-film sensing element for ozone, humidity and temperature, Sensors Actuators B Chem. 64 (2000) 42-48. doi:10.1016/S09254005(99)00481-5.

[13] I. a Ges, B.L. Ivanov, D.K. Schaffer, E. a Lima, A. a Werdich, F.J. Baudenbacher, Thin-film IrOx pH microelectrode for microfluidic-based microsystems., Biosens. Bioelectron. 21 (2005) 248-56. doi:10.1016/j.bios.2004.09.021.

[14] C. Da Viá, E. Bolle, K. Einsweiler, M. Garcia-Sciveres, J. Hasi, C. Kenney, et al., 3D active edge silicon sensors with different electrode configurations: Radiation hardness and noise performance, Nucl. Instruments Methods Phys. Res. Sect. A Accel. Spectrometers, Detect. Assoc. Equip. 604 (2009) 505-511. doi:10.1016/j.nima.2009.03.049.

[15] J. Park, S. Choi, S. Kim, Junction-tuned SnO2 nanowires and their sensing properties, J. Phys. Chem. C. (2011) 12774-12781.

http://pubs.acs.org/doi/abs/10.1021/jp202113x (accessed December 18, 2014).

[16] J.G.A. Brito-Neto, J.A. Fracassi da Silva, L. Blanes, C.L. do Lago, Understanding Capacitively Coupled Contactless Conductivity Detection in Capillary and Microchip Electrophoresis. Part 1. Fundamentals, Electroanalysis. 17 (2005) 1198-1206. doi:10.1002/elan.200503237.

[17] W. Olthuis, W. Streekstra, P. Bergveld, Theoretical and experimental determination of cell constants of planar-interdigitated electrolyte conductivity sensors, Sensors Actuators B Chem. 25 (1995) 252-256. http://www.sciencedirect.com/science/article/pii/0925400595850538 (accessed September 03, 2014).

[18] J. Hong, D.S. Yoon, S.K. Kim, T.S. Kim, S. Kim, E.Y. Pak, et al., AC frequency characteristics of coplanar impedance sensors as design parameters., Lab Chip. 5 (2005) 270-9. doi:10.1039/b410325d.

[19] V.F. Lvovich, C.C. Liu, M.F. Smiechowski, Optimization and fabrication of planar interdigitated impedance sensors for highly resistive non-aqueous industrial fluids, Sensors Actuators B Chem. 119 (2006) 490-496. doi:10.1016/j.snb.2006.01.003.

[20] P. Ben Ishai, M.S. Talary, A. Caduff, E. Levy, Y. Feldman, Electrode polarization in dielectric measurements: a review, Meas. Sci. Technol. 24 (2013) 102001. doi:10.1088/0957-0233/24/10/102001.

[21] H.P. Schwan, Four-Electrode Null Techniques for Impedance Measurement with High Resolution, Rev. Sci. Instrum. 39 (1968) 481. doi:10.1063/1.1683413. 
[22] S. Grimnes, Ø.G. Martinsen, Sources of error in tetrapolar impedance measurements on biomaterials and other ionic conductors, J. Phys. D. Appl. Phys. 40 (2007) 9-14. doi:10.1088/0022-3727/40/1/S02.

[23] B. a. Mazzeo, A.J. Flewitt, Two- and four-electrode, wide-bandwidth, dielectric spectrometer for conductive liquids: Theory, limitations, and experiment, J. Appl. Phys. 102 (2007) 104106. doi:10.1063/1.2815666.

[24] A. Nussbaum, Electromagnetic theory for engineers and scientists, Prentice-Hall, 1967.

[25] P. Mirtaheri, S. Grimnes, O.G. Martinsen, Electrode polarization impedance in weak $\mathrm{NaCl}$ aqueous solutions., IEEE Trans. Biomed. Eng. 52 (2005) 2093-9. doi:10.1109/TBME.2005.857639.

[26] N. Gavish, K. Promislow, Dependence of the dielectric constant of electrolyte solutions on ionic concentration, (2012). http://arxiv.org/abs/1208.5169v1 (accessed December 05, 2014).

[27] a Peyman, C. Gabriel, E.H. Grant, Complex permittivity of sodium chloride solutions at microwave frequencies., Bioelectromagnetics. 28 (2007) 264-74. doi:10.1002/bem.20271.

[28] Y. Nagasaka, A. Nagashima, Absolute measurement of the thermal conductivity of electrically conducting liquids by the transient hot-wire method, J. Phys. E Sci..... 1435 (1981). http://iopscience.iop.org/0022-3735/14/12/020 (accessed December $15,2014)$.

[29] J. Lyklema, Fundamentals of Interface and Colloid Science, Academic Press, 1995.

[30] D.K. Cope, The edge effect for planar electrodes, J. Electroanal. Chem. 439 (1997) 7-27. doi:10.1016/S0022-0728(97)00367-7.

[31] B. Timmer, W. Sparreboom, W. Olthuis, P. Bergveld, A. van den Berg, Optimization of an electrolyte conductivity detector for measuring low ion concentrations., Lab Chip. 2 (2002) 121-4. doi:10.1039/b201225a.

[32] B. a. Mazzeo, Parasitic capacitance influence of potential-sensing electrodes on four-electrode liquid impedance measurements, J. Appl. Phys. 105 (2009) 094106. doi:10.1063/1.3124365. 
Figures

(a)

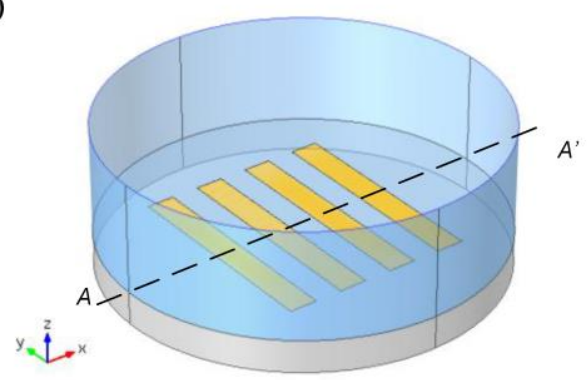

(c)

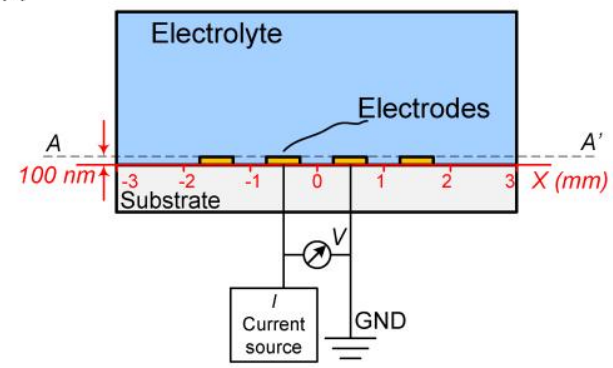

(b)

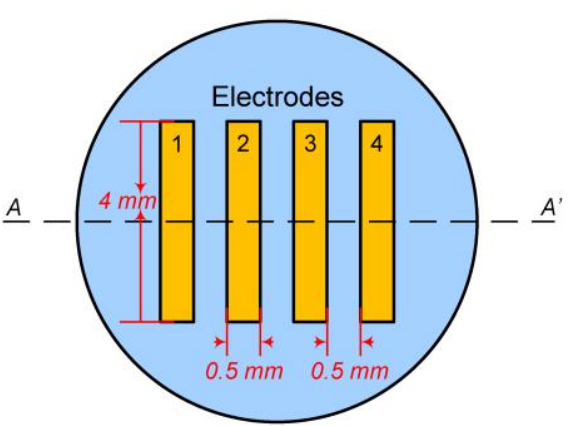

(d)

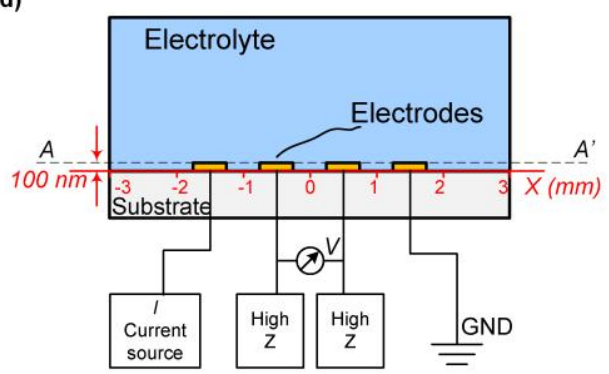

Fig.1 Schematic layout of the planar electrode systems and FEA simulation models. a) 3D view. b)

Topview, $\mathrm{Cr} / \mathrm{Au}$ double layer electrodes on glass substrates. c) Bipolar electrode arrangement. c) Tetrapolar electrode arrangement. 
(a)

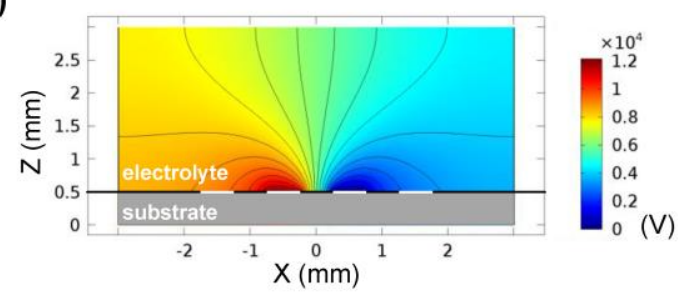

(c)

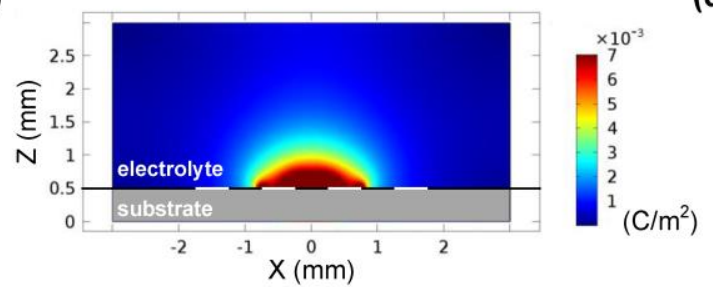

(b)

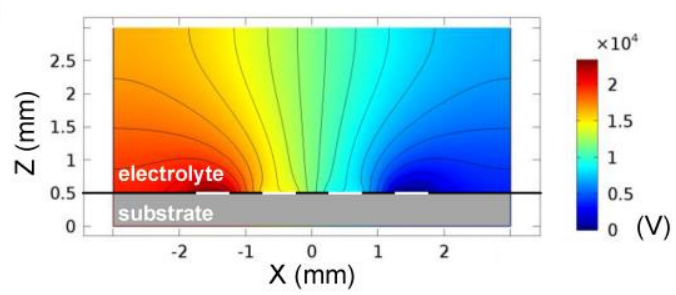

(d)

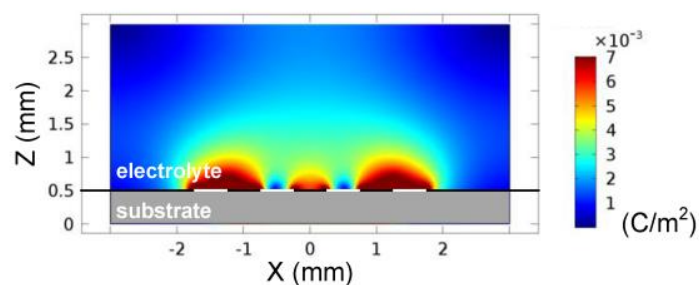

(e)

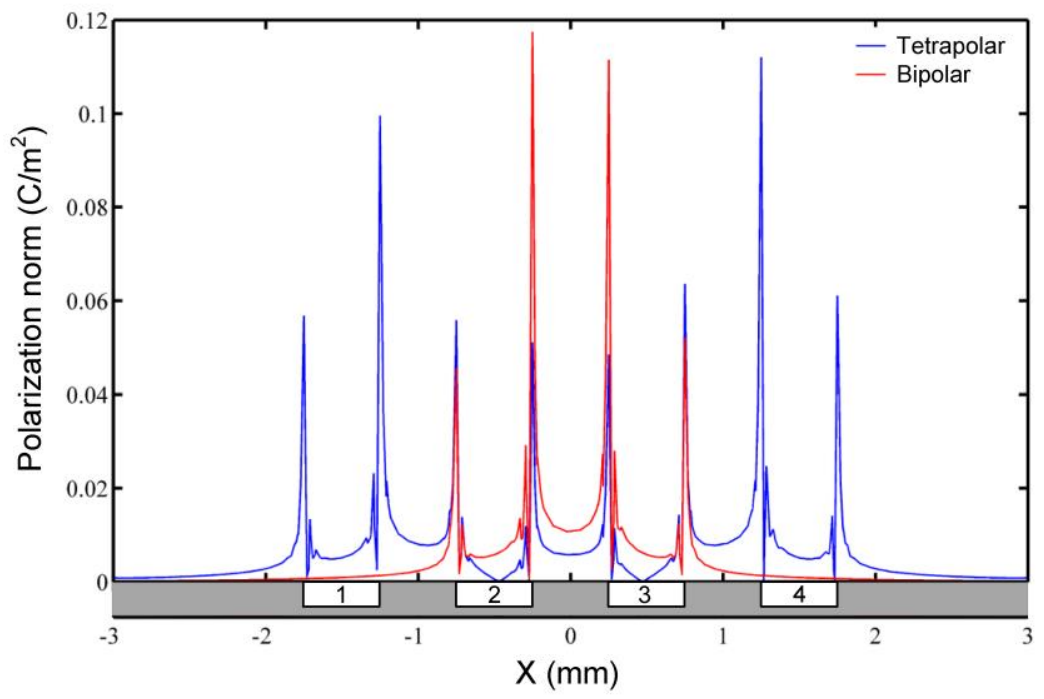

Fig.2 FEA simulation results for the bipolar and tetrapolar electrode arrangements. a) Cross-sectional electric potential distribution of the a) bipolar and b) tetrapolar electrode system. The color bar denotes the potential strength in V. Cross-sectional polarization distribution for c) bipolar and d) tetrapolar electrode arrangements. The color bar denotes the polarization strength in $\mathrm{C} / \mathrm{m}^{2}$. e) Normalized polarization over the electrode surface along the cut line (A-A') 

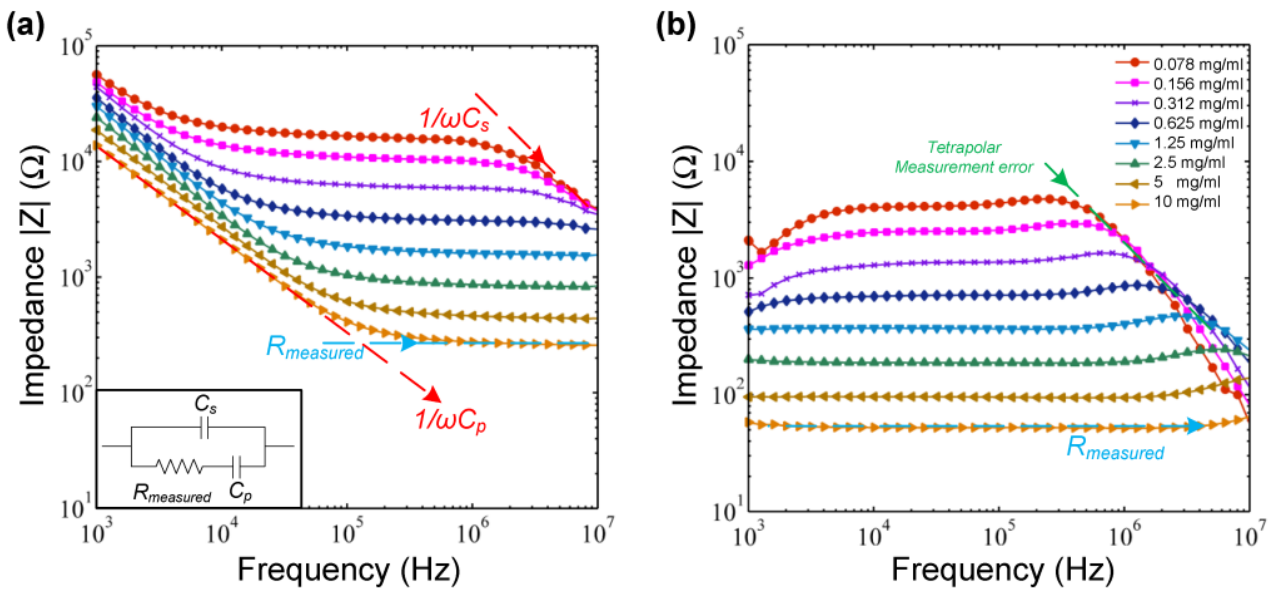

Fig.3 Impedance measurement results for different concentration saline samples with a) bipolar electrodes and b) tetrapolar electrodes. Subplot in a) is a typical equivalent circuit for the impedance with a solution capacitance $\left(\mathrm{C}_{\mathrm{s}}\right)$, a measured resistance $\left(\mathrm{R}_{\text {measured }}\right)$ and a polarization capacitance $\left(\mathrm{C}_{\mathrm{p}}\right)$

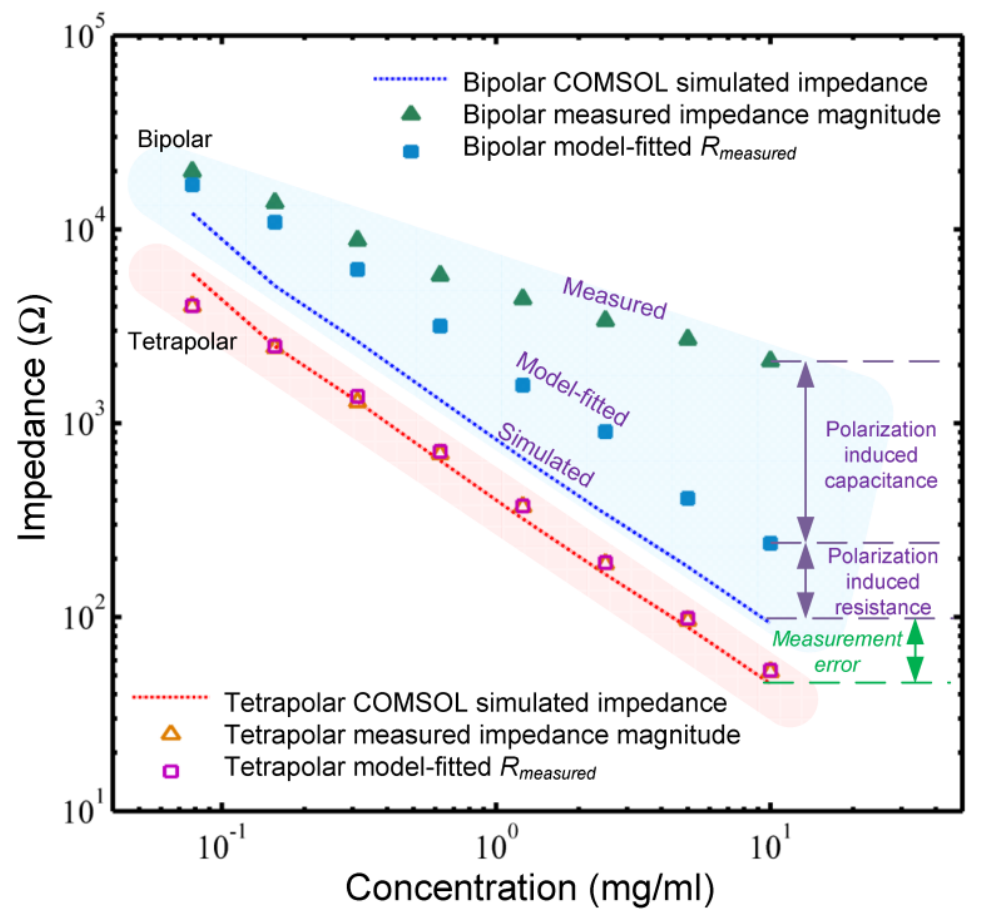

Fig. 4. Impedance of different saline concentrations for the bipolar and tetrapolar electrode systems. 


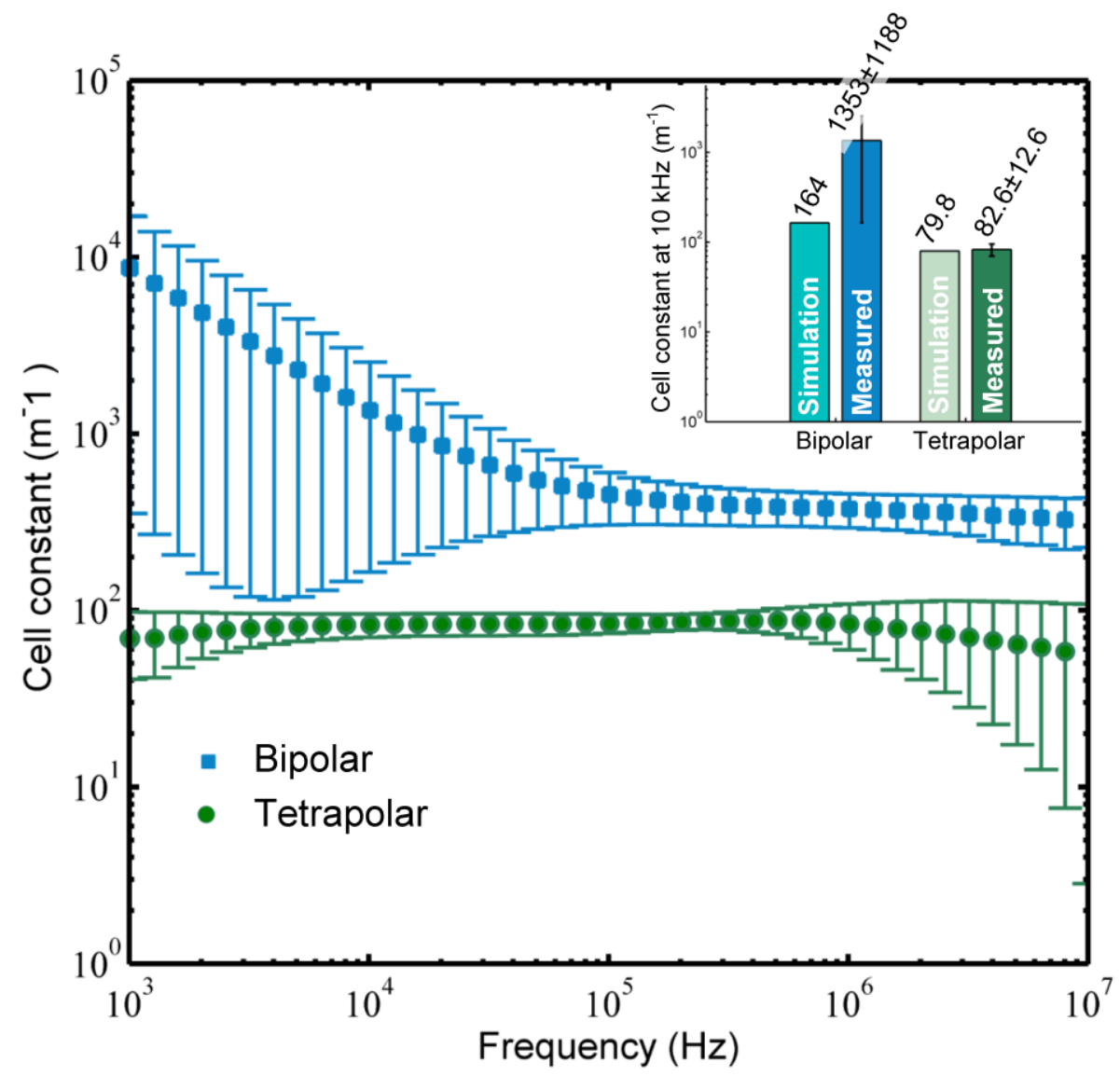

Fig. 5. Measured cell constants of the bipolar and tetrapolar electrode systems. Error bar shows the standard deviation over eight different sample concentrations as a function of frequency. Subplot shows the average cell constants over all frequencies. 